\title{
TDP2, TOP2, and SUMO: what is ZATT about?
}

Cell Research (2017) 27:1405-1406. doi:10.1038/cr.2017.147; published online 21 November 2017

Recently, ZATT (also known as ZNF451 or Zpf451) was reported by Schellenberg et al. to aid the removal of Topoisomerase II cleavage complexes by stimulating the phosphodiesterase activity of Tyrosyl DNA Phosphodiesterase 2. Although the full implication of this discovery is unknown, it will help us understand how cells respond to topoisomeraseinduced genome damage and chemotherapeutic topoisomerase 'poisons'.

Sources of endogenous DNA lesions are diverse, as are the biochemical mechanisms that comprise the DNA damage response (DDR) and remove them. DNA double strand breaks (DSBs) are potentially clastogenic and cytotoxic lesions, resulting not only from attack of DNA by electrophilic molecules such as reactive oxygen species but also from 'programmed' sources such as topoisomerase activity [1]. Topoisomerase II (TOP2) removes torsional stress from DNA during transcription and replication. To achieve this, it generates a transient DSB intermediate known as the 'cleavage complex' (TOP2cc), within which the topoisomerase is covalently linked to the 5'-termini of DSBs via $5^{\prime}$ phosphotyrosyl bonds. This enables intact DNA duplex to pass through the break and thereby decatenate DNA and remove DNA torsional stress [2]. While TOP2 normally reseals the DSB at the end of each catalytic cycle, under some conditions the cleavage complex becomes abortive, generating a potentially pathogenic DSB that requires DSB repair for removal.

The enzyme 5'-tyrosyl DNA phosphodiesterase 2 (TDP2) can initiate the re- pair of TOP2cc by hydrolytic removal of trapped topoisomerase peptide from the 5 '-termini of DSBs, thereby allowing the DSB to be religated [3]. The importance of this enzyme is illustrated by its proposed role in oncogenic translocations, resistance to chemotherapeutic topoisomerase 'poisons' and by causative mutations in the TDP2 gene in Spinocerebellar Ataxia Autosomal Recessive 23 (SCAR23) [4,5]. The denaturation and/or degradation of TOP2cc is thought to be a prerequisite for TDP2 activity, to enable access of the 5'-phosphotyrosyl bond within the active site of topoisomerase [6]. In the current work, Schellenberg et al.[7] challenge this model and clarify how TDP2 processes TOP2cc.

In contrast to previous models, Schellenberg et al. [7] identify a new TDP2dependent DNA repair pathway that involves TOP2 sumoylation independent of ubiquitination and proteasome activity. The authors show that TDP2 interacts with ZNF451 (aka Zpf451), a SUMO E3/E4 ligase/elongase that they denote ZATT (Zinc finger protein Associated with TDP2 and TOP2). ZNF451/ ZATT employs tandem sumo interacting motifs (SIMs) and a $\mathrm{C}_{2} \mathrm{H}_{2}$ zinc finger to promote SUMO2 conjugation and chain elongation in vitro, both in unperturbed cells and in perturbed cells treated with topoisomerase I poison, camptothecin [8]. ZNF451/ZATT was also identified as a TOP2-interacting protein by mass spectrometry, further implicating this protein in topoisomerase biology [9]. Schellenberg et al. identify a 'splitSIM' domain within TDP2 that confers SUMO2 binding specificity, and mutation of which ablates interaction with
TOP2 and ZNF451/ZATT.

Schellenberg and colleagues demonstrate that ZNF451/ZATT promotes both TOP2 sumoylation and TDP2 phosphodiesterase activity on intact TOP2cc. However, one of the most striking features of the work is that the latter stimulation occurs largely independently of SUMOylation, suggesting that direct interaction with ZNF451/ZATT alters the conformation of TOP $2 \mathrm{cc}$ in a way that renders the DSB more accessible to TDP2 (Figure 1). The role of SUMO in this pathway is more complex than postulated, as suggested by the surprising finding that mutation of the SUMO binding interface in TDP2 did not affect cell survival or DSB repair following etoposide treatment, even in the absence of proteasome activity [7]. In addition, this complexity is most likely further increased by the presence of a ubiquitin associated (UBA) domain located at the N-terminus of TDP2, which has been shown to interact with mono- and polyubiquitin chains [10].

A second striking feature of the work is that TDP2 appears to be the major if not only mechanism for removal of TOP2cc in the absence of proteosome activity. Loss of ZNF451/ZATT also leads to cellular hypersensitivity and delayed DSB repair following etoposide treatment in the absence of proteosome, albeit not to the same extent as loss of TDP2. Whilst ZNF451/ZATT thus appears to play a role in the response of cells to TOP2 perturbation, this may not be its primary function. Nevertheless, the identification of ZNF451/ZATT as a new player in the cellular response to topoisomerase inhibition raises new 


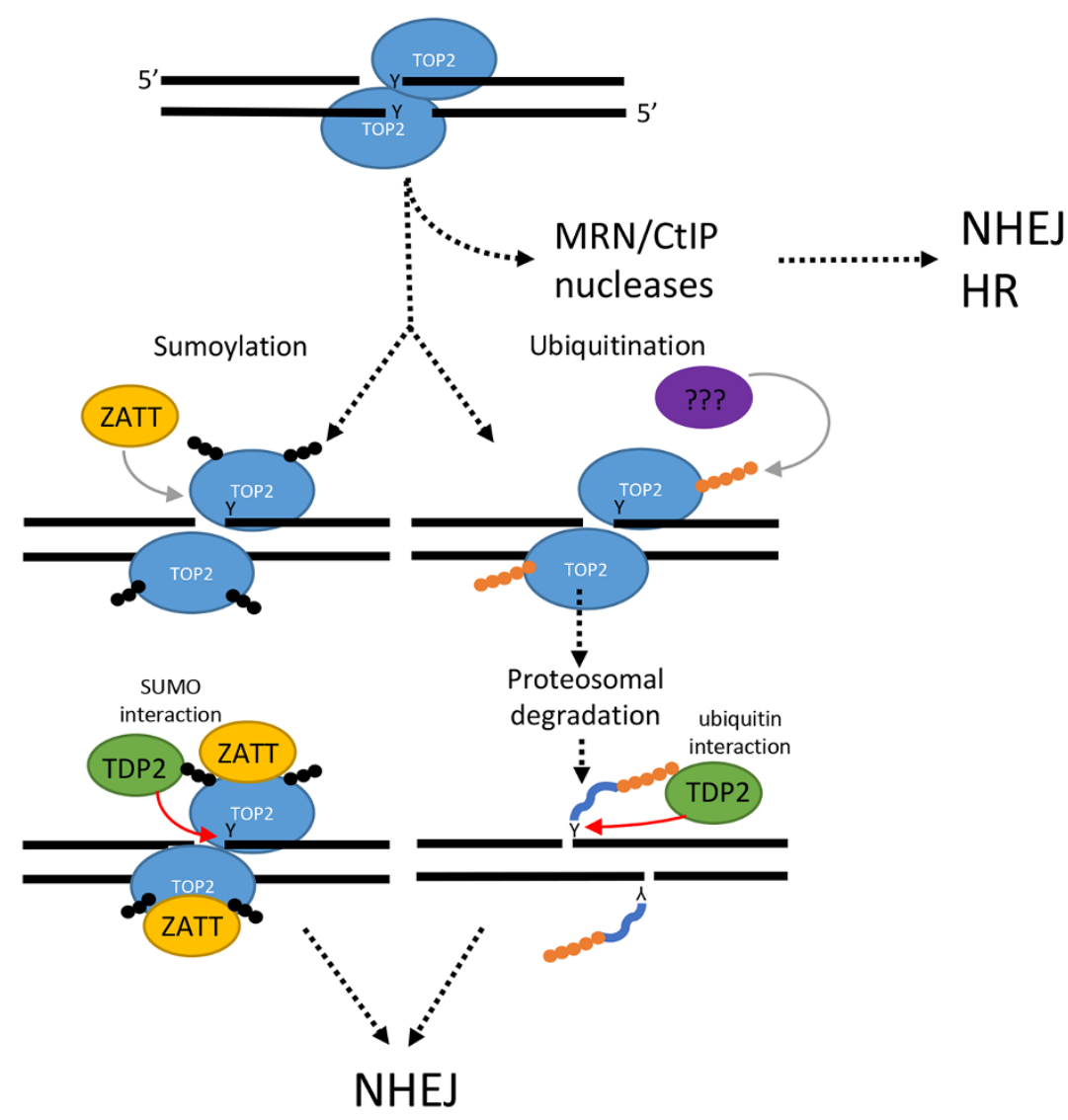

Figure 1 A model for the repair of abortive TOP2 cleavage complexes. Top, TOP2 becomes transiently linked to the 5 '-terminus of a DSB during its normal catalytic cycle via a phosphotyrosine bond, represented by the ' $Y$ ' on the DNA strand. Occasionally, these TOP2 cleavage complexes (TOP2cc) can become abortive, and thus require DSB repair for their removal. TOP2 can be removed from the 5 '-termini of the DSBs by a potentially mutagenic mechanism that employs nucleases such as MRE11/ RAD50/NBS1 (MRN) complex and CtIP. The DNA double strand break can then be repaired by homologous recombination (HR) or non-homologous end-joining (NHEJ). Alternatively, abortive TOP2cc can be repaired by TDP2-dependent mechanisms, providing an error-free route that does not require DNA degradation. Middle, the abortive TOP2cc is sumoylated by ZATT (left), and/or ubiquitylated (right). Bottom, TDP2 is recruited to the abortive TOP2cc by interaction with SUMO (left), and/or possibly ubiquitin (right), releasing TOP2 from the DSB by a hydrolytic mechanism. Note that the pathway on the left does not require proteosome activity, because ZATT and/or SUMO renders the phosphotyrosine bond accessible to TDP2. In contrast, the pathway on the right employs proteosome activity to render the 5'-phosphotyrosine bond visible to TDP2. Both TDP2-dependent pathways result in a DSB that can be ligated during NHEJ without nucleolytic processing, providing an error-free mechanism for DSB repair. Small black circles represents SUMO2 chains and orange circles represents ubiquitin chains.

questions concerning the role of SUMO with respect to cancer incidence, chemo therapy, and hereditary neurological diseases that are related to topoisomerase biology.

The work by Schellenberg et al. provides a fascinating new insight into topoisomerase biology and the cellular processes that regulate it. Like all exciting studies, the work raises more questions than answers. For example, what is the relative contribution of SUMO and ubiquitin in the processing of TOP2 cc, and how do cells choose which mechanism to employ to recognize, process, and repair these structures? The cell cycle could provide us with some clues, since TOP2 is reportedly heavily sumoylated during mitosis [11]. In any case, further examination of TOP 2 posttranslation modifications and their regulation will allow a better understanding of how cells protect their genome from topoisomerase-induced DNA damage.

\section{Guido Zagnoli-Vieira ${ }^{1}$,} Keith W Caldecott ${ }^{1}$

${ }^{I}$ Genome Damage and Stability Centre, University of Sussex, Falmer, Brighton, BN1 9RQ, United Kingdom

Correspondence: Keith W Caldecotta Guido Zagnoli-Vieira ${ }^{\mathrm{b}}$

${ }^{a}$ E-mail: k.w.caldecott@sussex.ac.uk

bE-mail: G.Zagnoli-Vieira@sussex.ac.uk

\section{References}

1 Tubbs A, Nussenzweig A. Cell 2017; 168:644-656.

2 Pommier Y, Sun Y, Huang SN, et al. Nat Rev Mol Cell Biol 2016; 17:703-721.

3 Ledesma FC, El Khamisy SF, Zuma MC, et al. Nature 2009; 461:674-678.

4 Gómez-Herreros F, Schuurs-Hoeijmakers JH, McCormack M, et al. Nat Genet 2014; 46:516-521.

5 Gómez-Herreros F, Zagnoli-Vieira G, Ntai I, et al. Nat Commun 2017; 8:233.

6 Gao R, Schellenberg MJ, Huang SY, et al. J Biol Chem 2014; 289:17960-17969.

7 Schellenberg MJ, Lieberman JA, HerreroRuiz A, et al. Science 2017; 357:14121416.

8 Eisenhardt N, Chaugule VK, Koidl S, et al. Nat Struct Mol Biol 2015; 22:959-967.

9 Uusküla-Reimand L, Hou H, SamavarchiTehrani P, et al. Genome Biol 2016; 17: 182.

10 Rao T, Gao R, Takada S, et al. Nucleic Acids Res 2016; 44:10201-10215.

11 Azuma Y, Arnaoutov A, Anan T, et al. EMBO J 2005; 24:2172-2182. 